\title{
De la Béatrice française de Berlioz à la Béatrice allemande de Richard Pohl : traductions interlinguistique et intersémiotique
}

\section{Charlotte Loriot}

Université Paris IV - Sorbonne

Inspired by Much Ado about Nothing of Shakespeare, the comic opera Béatrice et Bénédict of Berlioz countains a significant amount of irony. Richard Pohl, the author of the German translation, removed several strokes of irony for the performances in Weimar in 1863. This choice shows linguistic and cultural obstacles, as well as a specific aesthetic and ideological orientation. Irony, moreover, contributes to the different semiosis used in opera and circulates between text, music and theater. Music remains the same in both versions, but the new text changes the general atmosphere.

Béatrice et Bénédict fut créé à Bade (Baden-Baden) en 1862, puis à Weimar en 1863 dans une traduction allemande de Richard Pohl. Inspiré par Much Ado about Nothing de Shakespeare, l'opéra-comique de Berlioz contient une dose indéniable d'ironie, qu'il s'agisse du livret ou de la musique. L'ironie diffère toutefois entre l'original anglais, la version française et la traduction allemande.

Berlioz proposa d'abord à Peter Cornelius, auteur du Barbier von Bagdad, de réaliser la traduction de Béatrice et Bénédict. C'est finalement Richard Pohl, critique musical et écrivain allemand, qui en fut chargé. Dès 1855, Berlioz confiait à leur ami commun Liszt qu'il songeait à Pohl pour la traduction de ses Mémoires. Pohl traduisit d'autres écrits de Berlioz, les Grotesques de la musique et À travers chants, complétés dans une édition de 1864 par les Soirées de l'orchestre et le Traité d'instrumentation. Pohl se présente comme le seul journaliste allemand à avoir assisté à la création de Béatrice et Bénédict à Bade et il joua un rôle moteur dans l'organisation des représentations de Weimar en 1863, dans sa propre traduction.

Le rire et le sourire prennent des formes variées dans Béatrice et Bénédict, tantôt proches de l'humour et du comique, tantôt du grotesque, de l'ironie ou du pastiche satirique. L'ironie pose des problèmes de traduction, car elle repose sur les voies du renversement, de l'indirect et de l'implicite et rencontre des obstacles langagiers et culturels (sous-entendus à décrypter). Des enjeux esthétiques semblent également orienter la traduction de Pohl. La musique et le théâtre participant à l'ironie, on peut s'interroger sur l'influence que Pohl exerça sur l'atmosphère de l'œuvre. Quel pouvoir revient à la musique dans la création de l'ironie et quel est le pouvoir du traducteur? 


\section{Shakespeare, Berlioz et Richard Pohl : du texte aux livrets}

\subsection{Remaniements structurels de Shakespeare à Berlioz}

Lorsque Berlioz transforma Much Ado about Nothing en Béatrice et Bénédict, il se livra à une chirurgie radicale, qui fut commentée par Pohl dans un long article ${ }^{1}$. Berlioz ne conserva que sept des dix-huit personnages de Shakespeare, supprima l'intrigue menée par Don Juan, le frère de Léonato, et remplaça les personnages truculents de Dogberry et Vergès par le grotesque maître de chapelle Somarone - issu de Balthazar. Il maintint la double intrigue fondée sur les deux couples Claudio-Héro et BéatriceBénédict, conservant l'ironie et les joutes verbales associées à ces derniers. Comme il l'écrivit à son fils : «Je n'ai pris qu'une donnée de la pièce ; tout le reste est de mon invention. Il s'agit tout bonnement de persuader à Béatrice et à Bénédict (qui s'entre-détestent) qu'ils sont chacun amoureux l'un de l'autre et de leur inspirer par là l'un pour l'autre un véritable amour. » (Lettre à Louis Berlioz, 21 novembre 1860, Correspondance Générale (CG) VI, p. 74) L'opéra comporte d'autres dimensions que le comique : Berlioz ajouta des scènes pittoresques et de grands airs lyriques d'introspection (Héro et Béatrice).

Ces modifications eurent un impact sur l'ironie de l'œuvre, les cibles shakespeariennes étant détournées. Shakespeare attaquait les conventions sociales, la pratique du mariage et la bêtise des officiers municipaux. Les flèches contre le mariage visaient un monde de conventions que Béatrice et Bénédict refusaient de cautionner. Berlioz, lui, vise les conventions de l'opéra-comique, satirise sur la fugue musicale et se moque des compositeurs. Les personnalités semblent plus légères : le pittoresque et le sentimental sont privilégiés au détriment de la critique sociale. De Shakespeare à Berlioz, le rire est réadapté et redistribué différemment, notamment au profit d'une ironie ayant pour objet la musique (liée à Somarone). Les dialogues parlés intercalés tout au long de l'opéra-comique sont des adaptations très libres de Shakespeare, intégrant de nombreux ajouts, et les dimensions comique et ironique de l'œuvre originale s'en trouvent profondément transformées. On peut se demander si l'Allemand Pohl, qui connaissait le texte de Shakespeare, comprit les nouvelles cibles, implicites et françaises, de l'ironie berliozienne.

\subsection{Comparaisons des textes de Berlioz et de Richard Pohl : les tendances de la traduction}

La traduction de Pohl témoigne d'une grande liberté, se manifestant par des coupures ou des remaniements, ce qui était une pratique courante à l'époque. Il s'agit d'une adaptation plus que d'une traduction, enrichie 
d'épithètes poétiques apprêtées, parfois verbeuses. L'adéquation des paroles avec la ligne mélodique n'est pas toujours idéale, bien que Pohl se soit fait aider par deux autres traducteurs, Gustav Putlitz et Felix Mottl (qui participèrent aux Recitative, c'est-à-dire à certaines parties chantées, Pohl n'étant pas un musicien professionnel de formation). Analyser l'adéquation du texte avec la musique s'éloignerait de notre sujet, mais notons cependant que le rythme musical influence les choix du traducteur, qui doit respecter le nombre de notes des parties chantées. De fait, Pohl traduit plus librement les dialogues parlés.

Les lettres que Pohl adressa à Berlioz, certainement travaillées par leur auteur, comportent un certain nombre de fautes. Sa connaissance de la langue française n'était pas parfaite, et le premier problème qui se posa fut l'obstacle langagier. Certaines tournures ironiques semblèrent lui poser difficulté. Pohl en était conscient et n'hésita pas à demander conseil à Berlioz, par exemple pour À travers chants :

que signifie littéralement «à travers chants »? Est-ce un calembour avec, à travers champ? Je crains que ce titre n'est [sic] pas à traduire ; il faudra peut-être faire une «transcription » par exemple : «croisades musicales»? (Lettre de Richard Pohl à Berlioz, 12 octobre 1862, Correspondance Générale (CG) VI, p. 360).

Pohl semble avoir hésité sur le sens de certains calembours, jeux de langue prisés par Berlioz. Un exemple apparaît dès le Choeur $\mathrm{n}^{\circ} 1$ de Béatrice et Bénédict, lorsque le chœur s'exclame «Le More est en fuite », repris dans la scène suivante par Leonato. Le calembour, il est vrai, n'est pas très heureux. Mais Pohl ne choisit ni de coller au texte (der Mohr), ni de créer un effet équivalent, il remplace «Le More » par «Der Feind », et coupe le dialogue correspondant dans la scène $\mathrm{n}^{\circ} 2$.

Berlioz s'inquiéta dans une lettre à Liszt de l'incompréhension par Pohl des différents jeux de mots et sous-entendus :

Hier je t'ai envoyé en un paquet trois volumes manuscrits reliés, que je t'avais promis. [...] Il y aura là-dedans une foule de mots, d'allusions et de locutions tout à fait inintelligibles pour lui, mais je te prie de vouloir bien les lui expliquer. (10 mai $1855, \mathrm{CG} \mathrm{V,n^{ \circ }}$ 1965)

Béatrice et Bénédict comprend également une foule d'allusions ironiques. Béatrice, par exemple, interrompt ainsi le chœur d'entrée : «Assez ! assez ! aurez-vous bientôt fini de nous chanter: Gloire et victoire, Guerriers et lauriers ? Quelles rimes! Voilà les suites de la guerre !» Il s'agit d'une allusion aux chœurs d'introduction des opéras-comiques, devenus lieux communs. Outre cette pointe ironique, Berlioz se moque de la versification et du vocabulaire convenu souvent associés au genre. La traduction de Pohl, 
« Genug! Genug! Habt ihr das Lied noch nicht zum Überdruss gesungen? Und welche Reime! Lieder - wieder, schallen - hallen! (heftig) Ich halt' es nicht mehr aus!», limite l'attaque de Béatrice aux rimes, minimisant la portée des flèches.

Pohl ne semble pas toujours avoir compris les sous-entendus berlioziens. Par le biais de son personnage le maitre de chapelle Somarone, Berlioz tisse diverses allusions indirectes à des écoles musicales ou compositeurs de son temps. Selon le musicologue Joël-Marie Fauquet, Somarone caricature trois modèles: Spontini et sa vanité notoire; Cherubini avec le côté abstrait de la fugue ; Rossini, que Berlioz décrivait comme ne faisant que manger et boire et qu'il surnommait le « vieux satyre en retraite. » (Fauquet 2003, p. 62)

Le modèle spontinien apparaît notamment dans cette répartie de Somarone : "Mesdames et Messieurs, ... le morceau que vous allez avoir l'honneur d'exécuter est un chef-d'œuvre ! » (scène 11). Pohl ne la traduisit pas, mais en intégra un fragment plus loin, dans une scène qui n'existe pas sous cette forme dans l'original. De manière générale, Pohl ne se priva pas de couper certaines séquences et l'ironie berliozienne disparaît souvent. La vanité de Spontini était célèbre dans les années $1840^{2}$, mais il est vrai que le piquant de cette cible s'était émoussé en 1863, justifiant en partie les coupures de Pohl.

Le modèle de Cherubini, professeur et auteur de l'abstrait Cours de contrepoint et de fugue, semble également ignoré par Pohl. Somarone s'exclamait: "Le mot fugue veut dire fuite, et j'ai fait une fugue à deux sujets, à deux thèmes, pour faire songer les deux époux à la fuite du temps. » Ce raisonnement témoigne d'un sens poétique peu développé qui doit être pris au deuxième degré. L'exécution de l'Épithalame grotesque (en style fugué) donne ensuite lieu au dialogue suivant:

«Don Pedro. Bravo! c'est admirable. Musique symbolique! SOMARONE. Philosophique! Claudio. Cabalistique! BÉNÉDICT (bas) Et sudorifique, car il est en nage. »

Pohl supprima intégralement ce dialogue. La seule partie traduite qui s'en rapproche est la suivante :

(1) [Berlioz] Somarone. Ah! mon Dieu! vous me beuglez cet épithalame comme un De profundis! Vous ne comprenez donc pas... ce... ce chef d'œuvre ? ... Un chant de bonheur! un chant d'amour! qui doit ravir en extase les mariés... la nuit... qui doit s'envoler... s'exhaler... comme un parfum d'harmonie vers leur chambre nuptiale !

[Pohl] (zornig). Was soll das Schrei'en? Ihr weckt mit dem Spektakel ya die Todten, Und sollt den Lebenden Entzückung zeigen! 
L'ironie berliozienne visait également un autre modèle, le maitre de chapelle Barnabé, qui vantait son propre De profundis dans Le Maître de chapelle de Paër. Somarone est une caricature de Barnabé, dont Berlioz fustigea le ridicule dans un article de presse ${ }^{3}$. L'allusion est gommée par Pohl, qui supprime la référence au De profundis et à Barnabé, réduit celle à Spontini, et modifie le caractère du personnage.

Plus généralement, Berlioz ironise contre les fugues de la musique religieuse, dont il critique l'aspect conventionnel et peu inventif : la fugue créée par Somarone est une charge. Pohl ne peut couper le texte ridicule, puisqu'il est mis en musique: "Mourez tendres époux Que le bonheur enivre ! » devient «O Strib, du holdes Paar, von Liebesglück berauscht ! ». Malgré ces fautes volontaires de goût, dénoncées par le biais de l'ironie, Don Pedro est satisfait et félicite Somarone - nouvelle flèche, visant la musique officielle et les gouvernants aveugles, qui est supprimée par Pohl.

Une certaine sympathie de Berlioz pour Somarone, certes médiocre musicien, mais néanmoins bon bougre, râleur mais généreux, appréciant la bonne chère et n'hésitant pas à improviser un air à boire, apparaît ici. L'affection d'un Berlioz amusé se traduit notamment dans ce trait de Somarone (coupé par Pohl), devant la perplexité de Don Pedro : «Il est un peu... bourgeois, le général !». Cette phrase est une allusion implicite au mouvement Jeune France, à la jeunesse romantique des années 1830 menée par Victor Hugo ou Théophile Gautier.

Pohl supprime également la scène dans laquelle les domestiques sont furieux de devoir servir les musiciens (acte II, scène 1) :

PREMIER DOMESTIQUE. [...] N'est-ce pas une honte qu'il nous faille servir de tels misérables? DeUXIÈME DOMESTIQUE. Des soudars! Premier domestique. Des bohémiens. DeuXiÈme DOMEstiQue. Des gourgandines ! PREMIER DOMESTIQUE. Des joueurs de flûte !

Ces réparties sont à prendre au deuxième degré: Berlioz se moque indirectement de la manière dont les musiciens étaient considérés dans la société parfois rigide de son temps. On peut mentionner que le statut du musicien était autre à Weimar en 1863, mais Pohl ne sembla pas trouver la scène à son goût (et la coupa).

Les réparties ironiques traduites par Pohl jouent davantage sur des allusions internes à Béatrice et Bénédict. Lors du premier dialogue, Béatrice demande à un messager si «le seigneur Matamore» est de retour. Elle désigne Bénédict par cette allusion ironique à un poltron de commedia dell'arte, se targuant d'exploits jamais réalisés. Pohl traduit par «Signor Eisenfresser », transposition assez juste, qui permet de maintenir l'ironie.

De même, lorsque Bénédict s'exclame : «Dieu du ciel, faites-moi la grâce De ne pas femme m'octroyer, Blonde surtout! », il vise Béatrice. Le rire provient du jeu entre implicite et explicite, puisqu'on la reconnaît facilement. Pohl traduit alors assez littéralement l'ironie. Béatrice rétorque 
par une antiphrase : «Quelle menace ! ». Plutôt qu'une traduction littérale ( $\mathrm{O}$ welche Drohung», comme dans la traduction moderne de Bärenreiter), Pohl lui fait chanter : «Das darf ich nie verzeih'n », qui pose par ailleurs un problème d'adéquation au rythme musical. L'expression fonctionne comme antiphrase ironique, mais l'idée de pardon introduit un champ lexical plus lourd et connoté.

Le problème qui sembla se poser à Pohl, expliquant en partie ses remaniements, est l'allusion à un contexte qui n'était pas celui de Weimar. L'ironie fut possible à Bade car elle s'adressait à l'élite parisienne en villégiature, qui possédait la culture musicale sur laquelle ironisait Berlioz : il jouait sur la proximité avec ce public cultivé. On peut alors se demander si Pohl n'avait pas saisi les allusions, s'il préféra supprimer celles qui risquaient de ne pas porter, ou s'il obéit à d'autres enjeux. Il faut aussi noter que l'ironie des compositions de Berlioz est souvent moins fine que celle de ses écrits. Peut-être Pohl l'avait-il senti et l'évita-t-il pour cette raison. Mais comparons d'abord ses choix avec des traductions modernes.

\subsection{Libertés du traducteur}

Le traducteur doit-il être libre ou fidèle au texte? Les choix de Pohl interrogent sur ce que peut ou doit être une traduction. La manière dont il conçut son travail révèle des conceptions différentes des nôtres. Le volume La Traduction des livrets rappelle que la traduction était plus libre au $\mathrm{XIX}^{\mathrm{e}}$ siècle, en France comme en Allemagne : le traducteur ne se sentait alors nulle obligation de respecter le texte original à la lettre et l'adaptait à son goût personnel et au goût supposé du public. Transformer en profondeur un livret était monnaie courante.

On peut se demander comment Berlioz réagit, lui qui critiqua violemment les adaptations de Weber par Castil-Blaze. La correspondance entre Berlioz et Pohl au sujet de Béatrice et Bénédict est silencieuse sur ces questions, mais des échanges sont conservés pour À travers chants. Berlioz suggéra par exemple à Pohl de maintenir les citations de Shakespeare en anglais pour son feuilleton sur Roméo et Juliette, mais Pohl les traduisit en allemand (Lettre à Richard Pohl, 9 septembre 1859, CG VI, p. 23). Pohl demanda souvent son avis à Berlioz, mais ne le respecta pas toujours et déforma nombre de passages, tout en indiquant réaliser une traduction autorisée (autorisierte Übersetzung). Berlioz s'en irrite à propos de Béatrice et Bénédict dans une lettre à son ami Humbert Ferrand :

On me fait beaucoup d'éloges du travail du traducteur [Pohl]. Quant à moi je l'ai surpris, malgré mon ignorance de la langue allemande, en flagrant délit d'infidélité en maint endroit. Il s'excuse mal, et cela m'irrite. [...] N'y a-t-il pas de quoi pendre un traducteur ?... Mais 
quoi! il m'est si dévoué, c'est un si excellent garçon ! (11 avril 1863, CG VI, p. 427-428)

Berlioz émit donc des réserves sur le travail de Pohl, sans pour autant se fâcher avec lui. Ces libertés interrogent sur l'acte même de la traduction, qui va de l'équivalence à la création. L'un des pères fondateurs des normes modernes fut Richard Wagner, dans Opéra et drame (1852) - que Pohl connaissait, admirant l'homme, sa musique et fréquentant leur ami commun Liszt à Weimar. Wagner y critiquait violemment les normes existantes et fixa une série de règles à suivre, insistant sur la prosodie, la versification, les rimes, le phrasé, le rapport entre structures verbales, musicales et jeu des acteurs. Des normes traductives se sont développées au $\mathrm{XX}^{\mathrm{e}}$ siècle à partir de ces principes, aboutissant à des listes incluant la fidélité au message de l'original jusqu'aux contraintes liées aux structures musicales (Kaindl 2004, p. 43). Pohl ne déforma pas la musique de Berlioz, mais la chantabilité est parfois douteuse, et plus encore le respect du message.

À partir du moment où il prenait un parti-pris de liberté, Pohl pouvait soit transposer l'ironie berliozienne sur de nouvelles cibles, soit la supprimer - ce qu'il fit en général. Les choix des traducteurs modernes sont différents, à l'instar de Dagny Müller et Wulf Konold (Bärenreiter). Le ton s'éloigne des poncifs romantiques ou poétiques présents chez Pohl; la légèreté, le style conversationnel et la candeur réaliste de Berlioz, qui étaient gommées, réapparaissent. Dans l'ensemble, leur traduction reste neutre, littérale, proche du texte original. Parmi les phrases qui avaient été déformées ou coupées par Pohl, on peut citer «Le More est en fuite », qui devient «Der Mohr ist bezwungen » $\left(\right.$ Chøur $\left.\mathrm{n}^{\circ} 1\right)$, ou encore «Ah ! C'est un bel ouvrage» qui devient «Ach ja, es ist ein schönes Werk» (Somarone).

Dagny Müller et Wulf Konold introduisent parfois de nouvelles nuances ironiques, lorsque cela est possible sans s'éloigner de l'original. La phrase de Somarone «Il est un peu... bourgeois, le général ! d devient «Er ist wenig... bieder, unser General ». Dans cet exemple, les traducteurs ne choisisent pas les mots «bügerlich» ou «spiessig » (plus péjoratif), mais «bieder », qui signifie littéralement bon, brave, sans caractère - et évoque le mot connoté Biedermeier. De nouvelles allusions ironiques peuvent donc naître de la traduction («bieder» pour «bourgeois», ou encore «Eisenfresser » pour « Matamore» - comme le fit Pohl).

Une autre question se pose : s'il n'y a pas d'équivalent moderne aux allusions du compositeur, faut-il renoncer à toute transposition? Une solution radicale, souvent choisie pour respecter l'adéquation avec la musique, est de ne pas traduire. Une adaptation pose en outre le problème, outre l'adéquation au rythme musical, de devoir être constamment réactualisée. Les productions d'aujourd'hui préfèrent souvent réactualiser les œuvres voire les transformer en satires contemporaines, mais la tendance des choix éditoriaux, lorsque traduction il y a, est plutôt de rester 
neutre - ce qui permet de maintenir l'ironie alors comprise en fonction de la culture de l'auditeur. Ce type de traduction fait sourire uniquement les personnes qui connaissent la culture et ses codes, mais l'intrigue peut rester cocasse et la musique être suffisamment inspirée pour pallier la perte des allusions ironiques ou traits d'esprit. Pohl ne laissait pas cette possibilité.

Une traduction libre pourrait pourtant aboutir à une meilleure compréhension de l'ironie, en imaginant des équivalents allemands. Mais on note que Pohl la supprime dans l'ensemble. Pourquoi de telles modifications, allant même jusqu'à l'organisation du plan, alors qu'il serait plus facile de paraphraser? Il semble que d'autres raisons soient en jeu, qui dépassent les obstacles langagiers, culturels et la peur de n'être pas compris à Weimar. Sa traduction témoigne d'un parti-pris esthétique, qui fit barrage à l'ironie berliozienne.

\section{Du rire français à l'ironie allemande}

\subsection{Sphères géographiques et historiques de l'ironie}

Les scènes intégralement supprimées par Pohl sont précisément celles où la dimension ironique est importante (le dialogue de Somarone avec Don Pedro, Leonato et Claudio, ainsi que celui des deux domestiques). La conception allemande de l'ironie à l'époque de Pohl, bien différente de celle du milieu culturel français, explique peut-être ces choix.

Il n'existe pas vraiment de théorie spéculative du rire en France et rares sont les textes consacrés à ce sujet en dehors de Paul Scudo (Philosophie du rire, 1840), Baudelaire (L'Essence du rire, publication posthume), ou d'ouvrages plus tardifs de Bergson ou Jankélévitch. Les théories du comique musical se font plus rares encore, les écrits de Charles Beauquier demeurant assez marginaux. Des débats sur le sujet existaient néanmoins depuis le XVIII ${ }^{\mathrm{e}}$ siècle dans les journaux, soulevés à propos de comédies ou d'opéras-comiques: ces débats portaient généralement sur des questions de goût, s'interrogeant sur la distinction entre haut et bas comique et sur le juste ton propre à chaque genre. Certaines spécificités françaises semblent toutefois ressortir, en particulier l'art de la conversation et du bon mot ainsi que la dimension cinglante de l'ironie. Ces traits français sont très présents dans le livret de Béatrice et Bénédict, en particulier dans les dialogues opposant les deux héros éponymes. Ces séquences à l'ironie shakespearienne francisée contrastent avec d'autres sections moins fines comiques, grotesques ou humoristiques.

Comparée à la tradition allemande, l'ironie française reste proche du modèle rhétorique, liée à l'idée de raillerie et à la satire, privilégiant la notion de contraire. L'ironie dite romantique se développa en France sous forme d'apports de l'étranger combinés avec la tradition ironique française, 
celle de Pascal, Voltaire, La Fontaine et Rabelais. Selon Pierre Schoentjes (2001, pp. 120-129), l'ironie française, marquée par les traditions classique et rationaliste des Lumières, resta liée à la raison, tandis qu'en Allemagne elle se vit chargée des ambiguïtés qu'entraîne la scission de la personnalité en deux instances. L'ironie romantique allemande est plus sérieuse, alors que l'approche française coudoie la satire, le grotesque et évolue aux frontières du comique. Les écrits de Théophile Gauthier, que fréquentait Berlioz, en sont exemplaires. Berlioz, l'un des rares porte-parole du romantisme musical en France, est peut-être le musicien français qui fut le plus familier avec l'ironie romantique telle qu'elle était conceptualisée en Allemagne. Il s'en rapproche notamment dans la Damnation de Faust, dont la création parisienne reçut un accueil assez froid en dépit de l'effort d'acculturation à l'esthétique française - l'oeuvre fut en revanche plus rapidement appréciée en Allemagne. Mais Béatrice et Bénédict semble moins proche de cette forme d'ironie.

L'ironie romantique est en effet spécifique au XIX ${ }^{\mathrm{e}}$ siècle germanique et le contexte philosophique qui vit sa naissance est celui des frères Schlegel. Cette ironie se définit comme une attitude ludique de l'artiste par rapport à sa création, qui se distancie ironiquement de son œuvre. S'appuyant sur le paradoxe et la parabase, l'une des finalités de l'ironie est de montrer l'artifice. Elle propose un rapport réflexif à la création. Selon Friedrich Schlegel, l'imagination trouve son pendant indispensable dans l'ironie. Dans son alternance d'approbation et de négation, d'expansion et de contraction, d'autocréation et d'autodestruction, l'ironie devient le principe stimulant de la théorie romantique (Behler 1997, p. 86). Les esthétiques de l'ironie française et allemande ont donc peu en commun.

Les théoriciens de l'ironie s'accordent toutefois sur l'ambiguïté de l'ironie et sur son rapport à l'idéologie de groupes distincts. Le fonctionnement de l'ironie est conditionné par une communauté discursive : comme l'a montré Linda Hutcheon (1994), elle implique des relations dynamiques avec le contexte, la dimension sociale étant loin d'être négligeable. L'ironie se développe plus facilement lorsque les codes sont partagés : elle fait appel à une politique comme à une esthétique. Il reste à savoir si Pohl choisit délibérément de gommer l'ironie berliozienne, trop éloignée des conceptions allemandes.

\subsection{L'ironie de Béatrice et Bénédict analysée par Pohl}

Des écrits de Pohl nous permettent d'appréhender son regard sur Berlioz, en particulier dans sa correspondance ou ses écrits sur Bade et le compositeur. Dans son long article sur Béatrice et Bénédict, Pohl commence par décrire Berlioz lors de la création française de l'opéra-comique à Bade, le climat de l'œuvre et la simplification de l'intrigue shakespearienne ${ }^{4}$. Il précise que Berlioz s'adapta aux traditions parisiennes : 
L'opéra devait être comique; Berlioz a fait ici et là des concessions, qui ont résulté de sa fidélité à la tradition de l'opéra-comique parisien, avec dialogues parlés entre les numéros musicaux isolés ${ }^{5}$.

Suit une comparaison avec Wagner, dans laquelle Pohl montre que Berlioz a agi en régénérateur (Regenerator) plutôt qu'en réformateur (Reformator) comme il le fit dans d'autres œuvres antérieures. Pohl relève la liberté, la légèreté et l'humour de Béatrice et Bénédict, puis dépeint le caractère de chaque personnage : la grâce et le fin comique de Béatrice ( «Das Graziöse, Fein-Komische»), l'humour grossier et sec de Bénédict («das DerbHumoristische », «den trocknen Humor»), le grossier comique de Somarone (« die derbe Komik »), et le lyrisme sentimental d'Héro ( das Sentimental-Lyrische $»)^{6}$. Pohl ne mentionne pas l'ironie comme caractéristique générale, même si elle apparaît plus loin dans son analyse détaillée. Le premier emploi du mot ironie survient pour Somarone, à propos du dialogue parlé précédant la fugue de l'Épithalame grotesque :

Ici suit une scène d'un comique assez cru, dans laquelle Berlioz s'est fait plaisir de tout son cœur, et s'est vengé comme poète avec une ironie bien sentie de toutes les colères et fléaux qu'il a si souvent dû endurer comme compositeur et chef d'orchestre. ${ }^{7}$

Pohl emploie les qualificatifs d'un comique sec, mais efficace (« trocknen, aber wirsamen Komik ») pour Prilleur, la basse-bouffe chargée d'interpréter Somarone. Il qualifie de grotesque le texte de l'Épithalame et en propose une traduction, qui diffère de celle du livret de Weimar :

(2) [Berlioz] Chœur. Mourez tendres époux / Que le bonheur enivre ! / Mourez, pourquoi survivre / à des instants si doux.

[Article de Pohl: Neue Zeitschrift für Musik, 1862] Chor. O sterb, ihr holden Gatten, / Von Liebe ganz berauscht! / Ihr dürft nicht überleben / Der Stunde höchtes Glück!]

[Livret de Pohl: Weimar, 1863] Chor. O Strib, du holdes Paar / von Liebesglück berauscht! / Dies wär' der schönste Tod / den Ihr mit seinem tauscht.

Pohl décrypte les sous-entendus ironiques et traduit en allemand les justifications de Somarone sur le choix d'une fugue pour suggérer la fuite du temps, avec deux sujets pour représenter les époux (coupées en 1863). L'idée d'une fugue à programme est analysée comme une malice de Berlioz, dont la critique des fugues est connue depuis La Damnation de Faust. Pohl cite cette anecdote: «Berlioz m'expliqua en riant, qu'un contrapuntiste allemand vivant actuellement à Paris lui avait expliqué que cette fugue tout à fait correcte était le meilleur morceau de son opéra, dont 
il n'avait absolument pas remarqué l'ironie. ${ }^{8}$ Pohl utilise à nouveau le mot ironie, son analyse montrant qu'il est conscient des cibles implicites.

L'allusion à Spontini, par le biais de Somarone, est également relevée. Pohl traduit cette phrase, supprimée dans le livret : «Mesdames et Messieurs, le morceau que vous allez avoir l'honneur d'exécuter est un chef-d'œuvre!» («Meine Damen und Herren! Die Komposition, welche Sie jetzt auszuführen die Ehre haben, ist - ein Meisterwerk!»). Sa traduction est littérale. Parmi les modèles que vise l'ironie berliozienne, Pohl a donc décrypté au moins celui-là (seul ou aidé par Berlioz).

La lecture de cet article fournit des résultats inattendus : ce n'est pas suite à des incompréhensions que Pohl ne traduit pas toute l'ironie. Il avait deviné ou été informé des sous-entendus de Béatrice et Bénédict. Les « lacunes » de sa traduction sont des choix. Pohl exprima en outre à Berlioz combien traduire demandait de zèle et de temps (Lettre du 9 décembre 1862, CG VI, p. 378-380), ce qui est justifié quand on voit qu'il remania intégralement le texte, retraduisant différemment les extraits qu'il avait cités littéralement dans son article de 1862, et allant jusqu'à changer l'organisation et le contenu des scènes.

La traduction de Pohl élimina en outre la plupart des marqueurs qui aident à décrypter l'ironie : les signaux associés à Somarone sont gommés, l'ironie grotesque de la fugue n'est pas préparée. Berlioz était pourtant soucieux de la nécessité de ces indices, ayant déjà vu son ironie incomprise, et les théoriciens s'accordent pour montrer les risques de l'ironie, qui n'est pas toujours relevée en l'absence de marqueurs, c'est-à-dire de signaux permettant au récepteur de percevoir le double niveau de sens. Avec les suppressions de l'ironie et de ses indices opérées par Pohl, le comique, qui ne se rapproche pas davantage de l'ironie romantique, parait plus gratuit. Quelle était alors la finalité de cette traduction, donnant à penser que Pohl n'a pas compris l'ironie berliozienne?

\subsection{Les choix de Pohl dans le contexte culturel et esthétique de Weimar}

Traduire c'est interpréter. La version de Pohl révèle d'autres enjeux et modes de pensée; ces implicites idéologiques marquèrent sa traduction. Pohl s'adresse souvent à Berlioz avec une admiration sans bornes :

Cher et grand Maître! Vous recevez, j'espère ces lignes au 11 décembre - votre jour de naissance! je n'ai jamais oublié cette journée que j'ai célébrée une seule fois avec vous à Leipzig, il y a 9 années. [...] les 9 années d'Horace "ninum prematur in annum»se sont passées et ma vénération pour vous est restée invariable. (Lettre de Pohl à Berlioz, 9 décembre 1862, CG VI, p. 378) 
Cette admiration est orientée selon une esthétique spécifique. Pohl place Berlioz dans la filiation d'un Beethoven devenu légende, considéré comme un figure mythique du génie; il développe également l'idée d'une trilogie Berlioz-Liszt-Wagner, réformateurs de l'art et musiciens de l'avenir. Certains titres de chapitres de son livre consacré à Berlioz, qui prend la forme d'un recueil d'articles et essais, sont emblématiques: Moderne Programm-Musik, (1855), Die Symphonie seit Beethoven (1855), ou encore Berlioz's künstleriche Stellung zur Gegenwart (1856), chapitre dans lequel il compare Berlioz et Wagner. Le ton est donné dès le début de la préface :

Hector Berlioz, Franz Liszt, Richard Wagner, - ces trois noms constituent un rang étroit d'actes réformateurs, qui ont commencé avec la mort de Beethoven et se poursuivent jusqu'à présent et même au-delà $[\ldots]^{9}$

Cet état d'esprit marque l'ensemble de l'ouvrage. Pohl pose Berlioz en créateur de la Symphonie totale («Der Schöpfer der Total-Symphonie ») dans le chapitre sur son style (p. 22). Dans le chapitre sur Béatrice et Bénédict, il décrit Berlioz comme un Beethoven français, un génie, une personnalité toujours originale, une forte individualité. Le chapitre Berlioz als dramatischer Tondichter débute par une réflexion sur l'aspect double de l'œuvre d'art : la partie relative et la partie absolue, qui reste au-delà des contingences matérielles et temporelles (p. 120). Pohl est aussi marqué par l'idée de musique absolue, qui se développe à cette époque. Biographie et hagiographie, avec un vocabulaire parfois religieux, se confondent. Le $\mathrm{XIX}^{\mathrm{e}}$ siècle est justement une période où se constituent, depuis Beethoven, des figures d'hommes d'exception divinisés: le mythe du génie solitaire devient un lieu commun. La vie de Wagner, par exemple, témoigne d'un long travail de construction de son image. Liszt et Wagner se posèrent en «musiciens de l'avenir», ce que Berlioz critiquera. Pohl, ami de Liszt, semblerait avoir souhaité que Berlioz adhère à ce courant et les musiciens allemands partisans de la musique de l'avenir voyaient en Berlioz un des leurs, mais il s'opposa toujours à cette étiquette, refusant de se placer en porte-étendard. Berlioz rejetait globalement l'art wagnérien (notamment dans À travers chants, "Concerts de Richard Wagner. La Musique de l'avenir ») et s'éloigna de Liszt pour cette raison.

C'est à Weimar, l'un des bastions de Liszt et de la nouvelle musique, que se développèrent ces théories et que furent créés ou exécutés nombre d'opéras de Wagner. Richard Pohl comme les interprètes de Béatrice et Bénédict furent imprégnés de ce combat pendant les décennies 1840 et 1850 où Liszt fut Kapellmeister Extraordinaire (Huschke, 1982, pp. 116185). Pohl écrivit ainsi en 1853 un livre intitulé Akustische Briefe für Musiker und Musikfreunde, dans lequel il développe des conceptions métaphysiques, une esthétique cosmique, marquée par la philosophie de la nature (Naturphilosophie), par les courants philosophiques romantiques et 
idéalistes, et surtout par la musique de l'avenir. Berlioz en revanche, en dépit d'accents novateurs et romantiques, resta marqué par l'esthétique parisienne de son époque, c'est-à-dire par les catégories du joli, du bon mot, de la grâce ou du comique farcesque plus lourd, autant de catégories aux antipodes de l'ironie romantique allemande ou de Wagner.

Pohl se situe donc dans un autre courant esthétique et il était conscient de cette différence : comme il le note dans son article sur Béatrice et Bénédict, Berlioz n'est pas un idéaliste, car il ne renie pas le réalisme de ses origines françaises, même s'il tend de toutes ses forces vers le sentiment allemand de ses idéaux Gluck, Beethoven et Weber (p. 182). Pohl chercha à tirer Berlioz dans cette direction, quitte à modifier sa pensée. Il sembla en outre déçu, entre les lignes, de constater que Béatrice et Bénédict était moins réformatrice que d'autres œuvres. Il développa l'idée que Berlioz n'était pas reconnu en France à sa juste valeur, mais qu'il l'était en Allemagne, ce qui n'était pas faux, compte-tenu des échecs parisiens de Berlioz et du succès de ses concerts allemands ou des festivals organisés par Liszt à Weimar dès les années 1850. Pohl se sentit sans doute la «mission » de reprendre le flambeau en 1863, après le départ de Liszt. Béatrice et Bénédict arrivant comme une pirouette à la fin de la production de Berlioz, un retour ludique à la tradition après des œuvres délibérément novatrices, on peut supputer que Pohl, désorienté et peut-être même désappointé, chercha à orienter l'œuvre dans la direction qu'il aurait voulu que Berlioz choisisse.

Ses convictions influencèrent sa traduction. Pohl parait avoir été plus sensible aux sections faisant place à la nature romantique, à l'instar du Nocturne «Nuit paisible» (Héro et Ursule). Son commentaire est éloquent : «Le texte décrit dans une langue poétique le charme de la nature, qui se déploie aux yeux d'une mariée aimante, qui frissonne dans l'attente de son bonheur. [...] ${ }^{10}$ La traduction du livret, qui accentue encore les poncifs poétiques, reste relativement fidèle. Pohl développa le dialogue introductif, à l'inverse des scènes comico-ironiques drastiquement réduites. Berlioz était adepte du mélange des tons, des styles et des genres, mais l'œuvre de Pohl, ainsi modifiée, devient plus uniforme.

Il semble que Pohl ait considéré les flèches satiriques et réalistes de Berlioz comme inadéquates à son projet esthétique, inadaptées à l'ironie cosmique dans la lignée des théories romantiques de Schlegel. Il chercha à gommer la partie «relative» de l'art, pour ne développer que la partie « absolue ». De la même manière que Berlioz s'était adaptée à l'esthétique parisienne, Pohl s'adapta à l'esthétique allemande telle qu'elle avait été définie par Liszt et son entourage wagnérien. Le Duo ${ }^{\circ} 4$ est emblématique de cette orientation. Il ne put être coupé, puisqu'il était mis en musique par Berlioz, mais quelques choix traductifs orientent le sens dans une nouvelle direction : 
(3) BÉNÉDICT. Aimable dédain! on est trop heureux D'endurer vos coups. Que ne suis-je maitre De suivre vos pas! Qui, tant qu'ici bas Vous daignerez paraître Pour charmer nos yeux, Qui donc voudrait aller aux cieux ? BÉATRICE. J'ai pitié de votre ironie. BÉNÉDICT. Moi railler! certes, je le nie. Mais, franchement, non, vous avez raison. [...]

BENEDIKT. Mein Fraülein, Ihr fordet zum Rampf mich auf's neue, mit altem Uebermuth? Nur zu! Ich bewies, dass ich Zungen nicht scheue, Auch damit fecht' ich gut, Ich sehnte oft so sehr Nach Euch mich wieder her! Wenn er uns erscheint Mit Schönheit geschmückt, Die Feben entzückt: Bermeidet man nimmer den Feind! BEATRICE. Feig fucht Ihr, Mit Schmeichelei zu siegen? BENEDIKT. Schmeicheln? Ich? Dann müsst' ich unterliegen! Doch - sei es bekannt - Ich war zu galant ; [...]

Le mot «ironie », présent chez Berlioz, est transformé par Pohl en «schmeichelei» (flatterie). Le surnom de Béatrice «Aimable dédain» devient « Mein Fraülein » et Pohl ajoute le mot «galant». Il réduit donc les échanges des deux héros à la galanterie française, supprimant le piquant de certaines réparties et refusant le mot ironie, qui lui semble probablement trop éloigné de la conception allemande. Pohl connaissait les écrits de Berlioz, comprit son ironie, mais ses enjeux étaient différents (musique de l'avenir, musique absolue).

Les flèches de Berlioz contre le mariage étaient personnelles, après deux expériences difficiles : il décrit le mariage comme « la chaîne la plus lourde qui se puisse porter » (Lettre à son fils du 14 février 1861, CG VI, p. 199.). Les piques de Béatrice et Bénédict avaient une dimension de vécu ; en les tranformant, Pohl gomme la partie relative de l'art. La légèreté et l'humour n'en restent pas moins présents dans ce duo - la présence de contributeurs pour les sections musicales, même supervisés par Pohl, ayant peut-être contribué à y maintenir davantage d'ironie.

De manière générale, tout traducteur peut choisir de rester proche de la culture de départ, ou d'adapter l'œuvre à son nouveau contexte, ce que fit Pohl. Ses choix traductifs remanient la culture de départ vers celle d'arrivée (la musique de l'avenir), pour laquelle il modela son travail. Il est douteux que Pohl ait supprimé les traits ironiques par crainte de les voir incompris à Weimar: il aurait plutôt cherché à rajouter de nouveaux indices ou transposé l'ironie à de nouvelles cibles. Son esthétique semble avoir orienté sa traduction et constitué un nouvel enjeu : l'ironie berliozienne lui semblait sans doute trop éloignée à la fois de l'ironie romantique, de la musique absolue et de la musique de l'avenir. 


\section{L'ironie entre texte, théâtre et musique}

\subsection{Une ironie intersémiotique}

La distinction établie par Roman Jakobson entre traduction intralinguistique (reformulation), interlinguistique (d'une langue à l'autre) et intersémiotique (transmutation), est connue (1963, p. 78-86). La traduction d'un livret d'opéra possède une dimension interlinguistique, mais aussi un niveau intersémiotique. Dans Béatrice et Bénédict l'ironie verbale est traduite dans la musique et le théâtre. Les rapports entre ces trois niveaux sont complexes: la traduction ne fonctionne pas à sens unique du livret aux autres sémioses.

On peut se demander comment fonctionne l'ironie musicale. L'ironie possède une dimension fondamentalement langagière et la plupart des traits ironiques de Berlioz appartiennent aux dialogues parlés, mais ils se manifestent aussi dans la musique, selon plusieurs modalités. On peut d'abord distinguer les séquences où l'ironie accompagne les réparties, par exemple lors de la joute spirituelle entre Béatrice et Bénédict $\left(\right.$ Duo $\left.\mathrm{n}^{\circ} 4\right)$ et dans le Trio masculin $\mathrm{n}^{\circ} 5$. Dans ces numéros, la musique ne crée pas de sous-entendus ironiques: elle se contente d'accompagner le texte, d'une manière vive et piquante. Berlioz emploie un lexique musical qui génère une légèreté, avec notes piquées, trilles, traits rapides, notes répétées, ou encore une orchestration légère. La musique illustre en quelque sorte l'effet de l'ironie, incarnant son piquant, sa verve et son côté alerte.

D'autres effets ironiques apparaissent lorsque la musique se distancie du texte. À la fin du Trio $\mathrm{n}^{\circ} 5$, par exemple, Bénédict consent à voir inscrit sur son toit «Ici l'on voit Bénédict l'homme marié », s'il accepte un jour d'épouser une femme. Le rythme enlevé s'interrompt alors subitement pour laisser place à un récitatif dans le grand style, qui ne saurait être pris au sérieux dans ce cadre. Berlioz vise implicitement le genre noble dans un pastiche qui peut s'interpréter de manière à la fois ludique (proche de l'humour) ou satirique (vers l'ironie). Ce choix dépend aussi des musiciens, qui peuvent orienter l'interprétation dans un sens ou l'autre. Cette ironie fonctionne encore par rapport au texte, mais introduit de nouvelles cibles et un niveau de sens supplémentaire.

L'ironie peut aussi naître de la circulation entre les trois niveaux. Le texte n'est pas destiné à être seulement lu et l'ironie peut résulter de la coopération entre texte, musique et théâtre. Dans le Duo $\mathrm{n}^{\circ}$ 4, par exemple, lorsque Bénédict prie Dieu de ne pas lui octroyer de femme, «blonde surtout », une didascalie précise «montrant Béatrice ». L'allusion implicite à un mariage avec Béatrice est donc indiquée par le geste - la didascalie étant supprimée par Pohl. La musique surenchérit, comme dans le Trio $\mathrm{n}^{\circ} 5$ déjà cité, par un pastiche, mi-humoristique, mi-ironique, des prières, devenues un lieu commun de l'opéra-comique. Autre exemple de 
coopération : lorsque les hautboïstes jouent faux (scène 11), Somarone, dont le nom signifie «gros âne », s'exclame «Il y a de quoi déchirer des oreilles d'ânes », tout en "portant la main à ses oreilles »(didascalie). Chacun des trois langage participe à sa façon dans cet exemple à la création du rire ou du sourire.

Berlioz développe aussi une ironie plus musicale dans l'Épithalame grotesque. L'ironie résulte de la condamnation implicite du style fugué par Berlioz, qui le tourne en dérision. Comme dans le Duo $\mathrm{n}^{\circ} 4$ et le Trio $\mathrm{n}^{\circ} 5$, il s'agit d'une citation stylistique, entre pastiche et charge, mais le support textuel joue un moindre rôle. Le texte ridicule contribue à l'ironie, mais elle aurait aussi fonctionné sur des paroles neutres (comme le mot Amen de $\mathrm{La}$ Damnation de Faust). Le texte joue ici avant tout un rôle de marqueur, qui introduit l'ironie. Le texte ne serait pas nécessaire pour le public, certes très réduit, de mélomanes ou musiciens connaissant les valeurs de Berlioz. Certains moments de musique instrumentale, tels l'ouverture avec sa musique vive et piquante, pourraient même être analysées comme de l'humour ou de l'ironie purement musicale.

Berlioz joue donc sur la circulation entre les différentes sémioses, l'ironie pouvant naître d'une coopération ou d'un rapport complémentaire. La finalité de l'exécution, associée aux difficultés posées par la musique (rythme, accentuation, musicalité et chantabilité de la traduction), implique un savoir-faire particulier pour traduire un livret. C'est sans doute la raison pour laquelle Berlioz avait d'abord pensé à un compositeur, Peter Cornelius, avant de s'adresser à Pohl. Pohl n'était pas professionnel : il lisait la musique et avait étudié la partition, mais était d'abord écrivain et critique musical. Son statut explique certains de ses choix et le côté idéologique de la traduction. Pohl écrivain défendait une esthétique spécifique : il fit œuvre de créateur lorsqu'il adapta les textes de Berlioz.

\subsection{L'interprétation des acteurs allemands}

La musique resta la même lors de la création allemande à Weimar, mais les acteurs jouaient un nouveau texte, moins ironique. Quelles furent les incidences sur leur interpréation et sur la perception du public ?

Dans sa correspondance rédigée lors de son séjour à Weimar en avril 1863, Berlioz décrit le succès de l'œuvre, insistant sur la réussite des sections introspectives ou sentimentales. Son seul reproche, récurrent, est le suivant: «la Béatrice ( $\mathrm{M}^{\mathrm{me}}$ Milde) ne peut pas s'italianiser, elle reste une Béatrice allemande et sentimentale. » (Lettre à H. Lecourt, 7 avril 1863, CG VI p. 421); ou encore $" \mathrm{M}^{\mathrm{me}}$ Milde est charmante mais elle a fait de la lionne sicilienne une Béatrice allemande, et ses yeux de colombe ne parviennent pas à darder des flammes. C'est un autre genre de vérité. » (Lettre à P. Viardot, 9 avril 1863, CG VI p. 425). Mais Berlioz sembla dans l'ensemble ravi, y compris de Béatrice, narrant à son ami Humbert Ferrand 
qu'il n'hésita pas à l'embrasser, tout à sa joie, à la grande surprise de l'interprète (11 avril 1863, CG VI, p. 428). Berlioz décrit en particulier le succès du trio de femmes :

Le succès de Béatrice a été flambant, l'exécution excellente dans son ensemble. Le grand-duc et la grande-duchesse et la reine de Prusse m'ont accablé de compliments. La reine surtout m'a dit des choses, oh! mais des choses que je n'ose vous répéter. Le morceau qu'elle aime le plus, c'est le trio des trois femmes, tout en avouant que le duo est une invention ravissante, et que l'air de Béatrice et la fugue comique lui plaisent infiniment. (Lettre à Lambert et Aglaé Massart, 9 avril 1863, CG VI, p. 424)

La Neue Zeitschrift für Musik publia un article d'Heinrich Borges sur l'événement (17 avril 1863, p. 135-136), qui commence par une comparaison avec les réformes wagnériennes, montrant que Berlioz a respecté des conceptions plus anciennes de l'opéra. L'auteur inscrit Berlioz dans une filiation beethovenienne (comme Pohl), en comparant Béatrice et Bénédict avec Fidelio. Il s'interroge sur la compatibilité entre la grâce française et Shakespeare et conclut que les moments où l'on est touché par la ferveur et la profondeur, qui constituent le cœur de l'âme allemande, ne sont pas rares, comme le Nocturne. Il reconnaît le travail de Pohl :

Il faut à cette occasion mentionner avec une reconnaissance particulière l'exquis arrangement du texte français par R. Pohl. Il surpasse l'original français en valeur poétique, et Pohl a beaucoup à s'attribuer pour avoir encore élevé la riche conception poétique. ${ }^{11}$

Le journaliste note que l'interprète de Béatrice orienta son rôle vers le poétique. Il relève également la vivacité de Bénédict et le comique cru (drastisch) de Somarone. Les modèles de Somarone étant gommés par la traduction, on comprend que l'ironie moins crue ne soit pas relevée. Il faut aussi rappeler que Rosa von Milde était plus familière du répertoire et du style wagnériens, dont elle fut l'une des premières grandes interprètes, créant notamment le rôle d'Elsa dans Lohengrin ${ }^{12}$.

Il est probable qu'Heinrich Borges ait traduit une perception générale et que le travail de création de Pohl ait été applaudi comme tel à Weimar. Pohl ne s'était donc pas trompé sur le succès de sa traduction, lorsqu'il prit la liberté d'adapter l'œuvre. Berlioz sembla de son côté satisfait: "En somme, c'est beaucoup mieux qu'à Bade. » (Lettre à Pauline Viardot, 9 avril 1863, CG VI p. 425) L'ironie était pourtant moins présente, avec l'interprétation poétique de la nouvelle Béatrice. La chanteuse respecta son texte, mais Berlioz ne parlant pas allemand, il ne réalisa probablement pas la portée et l'incidence des modifications de Pohl sur son jeu. L'impact de 
la traduction fut donc assez fort et donna lieu à "un autre genre de vérité », dans lequel l'ironie avait moins de place.

La musique resta inchangée, mais la force sémantique du texte fut prépondérante. L'accompagnement demeura vif et léger dans les joutes ironiques de Béatrice et de Bénédict, mais ne généra pas d'ironie à lui seul ; les pastiches ludico-ironiques furent maintenus, sans être considérés comme un trait essentiel. Seule l'ironie de la fugue semble avoir été relevée, mais Berlioz l'avait sans doute explicité lors de discussions privées. La musique dépasse les frontières linguistiques, mais elle en reste tributaire dans l'opéra, où la force sémantique des paroles est forte. La traduction de Pohl révéla d'autres enjeux esthétiques: l'ironie fut moins présente dans l'exécution de cette nouvelle œuvre.

Outre les obstacles culturels, linguistiques et esthétiques, l'éloignement dans le temps est un autre facteur qui réduit la portée de l'ironie. Les cibles passent et l'ironie se périme. Les exécutions de Béatrice et Bénédict reposent d'ailleurs le plus souvent aujourd'hui sur une version modernisée du livret, qui modifient à la fois les cibles caduques et les sections comiques moins fines et moins réussies. Pohl, qui distinguait dans ses analyses la valeur relative et la valeur absolue de l'art, avait sans doute relevé que les flèches ironiques de Berlioz ne seraient pas éternelles et qu'elles demanderaient rapidement modernisation et adaptation. L'ironie rattache l'œuvre à son contexte et Pohl voulait au contraire l'en détacher afin d'élever Berlioz au niveau des œuvres absolues et éternelles. Pohl fit un choix traductif, celui de supprimer ce qui est contingent et ne peut être modernisé, plutôt que de le transposer éternellement.

\section{Conclusion}

L'ironie peut être exprimée par les trois sémioses mises en œuvre dans l'opéra, le verbe, la musique et le théâtre, mais c'est toutefois le langage verbal, plus directement significatif, qui semble dominer. S'il existe une circulation entre les trois modes d'expression, la sémiose verbale commande le sens général. La musique pure véhicule certes l'ironie, mais en présence d'un livret, l'auditeur se concentre sur le sens transmis par le texte; le livret prime également sur la musique dans le choix des jeux scéniques par les acteurs. Le livret de Pohl orienta la direction esthétique des représentations de Weimar et la dimension ironique de la musique de Béatrice et Bénédict resta tributaire du verbe: lorsque le texte change, l'auditeur se focalise sur sa nouvelle signification. Le pouvoir du traducteur d'opéra est donc lourd de conséquences. 


\section{Références}

Adam, A. (1996). Lettres sur la musique française (1836-1850). Genève : Éditions Minkoff.

Behler, E. (1997). Ironie et modernité. O. Mannoni (traduit en français. Original allemand Ironie une literarische Moderne, 1997). Paris : Presses Universitaires de France.

Berlioz, H. (1864). A travers chants. R. Pohl (traduit en allemand. Original français $A$ travers chants, 1862). Leipzig : von Gustav Heinze.

Berlioz, H. (1863). Beatrice und Benedict. R. Pohl (traduit en allemand. Original français Béatrice et Bénédict, 1862). Weimar: Tantz.

Berlioz, H. (1870). Beatrice und Benedict. Richard Pohl, G. zu Putlitz \& F. Mottl (traduit en allemand. Original français Béatrice et Bénédict). Berlin: Bote \& Bock.

Berlioz, H. (2003). Beatrice und Benedict. D. Müller \& W. Konold (traduit en allemand. Original français Béatrice et Bénédict). Kassel: Bärenreiter.

Berlioz, H. (1995). Correspondance Générale VI, 1859-1863. P. Citron (Ed.). Paris : Flammarion.

Cairns, D. (2002). Hector Berlioz. Servitude and greatness. 1932-1869, vol. 2. D. Collins (traduit en français. Original anglais Hector Berlioz. Servitude et grandeur. 1932-1869). Paris : Fayard.

Citron, P., \& Reynaud, C. (Eds.) (2003). Dictionnaire Berlioz. Paris : Fayard.

Farina, S. (2005). La traduction des livrets d'opéra au XIX siècle, thèse, Université de Paris Sorbonne.

Fauquet, J.-M. (2003). Somarone ou l'ivresse de soi, L'Avant-Scène Opéra 214 : Béatrice et Bénédict, 60-63.

Jakobson, R. (1963). Des aspects linguistiques de la traduction, In Essais de linguistique générale (pp. 78-86). N. Ruwet (traduit en français. Original anglais On Linguistic Aspects of Translation). Paris : Les Éditions de Minuit.

Jankélévitch, V. (2005). L’ironie. Paris : Flammarion.

Huschke, W. (1982). Music im klassischen und nachklassischen Weimar 1756-1861. Weimar: Hermann Böhlaus Nachfolger.

Hutcheon, L. (1994). Irony's edge: The theory and politics of irony. London: Routledge.

Kaindl, K. (1995). Die Oper als Textgestalt, Tübingen : Stauffenburg.

Marschall, G. R. (Ed.). (2004). La traduction des livrets: Aspects théoriques, historiques et pragmatiques, Paris : Presses de l'Université Paris-Sorbonne.

Pohl, R. (1853). Akustische Briefe für Musiker und Musikfreunde: Eine populäre Darstellung der Musik als Naturwissenschaft in Beziehung zur Tonkunst, Leipzig: von Bruno Hinze.

Pohl, R. (n.d.). Baden-Baden et ses environs. C. Cayard (Traduit en français. Original en français). Münich: A. Bruckmann.

Pohl, R. (1884). Hektor Berlioz: Studien und Errinungen. Leipzig: B. Schlicke

Schoentjes, P. (2001). Poétique de l'ironie. Paris : Seuil.

Stricker, R. (2003). Berlioz dramaturge. Paris : Gallimard. 
Wagner, R. (1982). Opéra et drame. J.-G. Prod'homme (Traduit en français. Original allemand Oper und drama). Paris : Éditions d'aujourd'hui.

1 L'article de R. Pohl sur Béatrice et Bénédict fut publié dans la Neue Zeitschrift für Musik en trois parties, le 28 novembre et les 5 et 12 décembre. Il fut repris en 1884 dans son Hektor Berlioz sous le titre "Beatrice und Benedikt in Baden-Baden", pp. 176-200. Sur Richard Pohl, voir notamment la notice réalisée à son nom par John Warrack et James Deaville dans le Grove Music online.

2 Voir les Lettres d'Adolphe Adam au journaliste berlinois Samuel Spiker.

3 Critique de Berlioz sur Le Maître de chapelle publiée dans Le Rénovateur, le 27 juillet 1834 : Critique musicale, Paris, Buchet-Castel, vol. 1, p. 309.

4 «Beatrice und Benedikt in Baden-Baden », op. cit.

5 «Wenn er das ganze Shakespearesche Drama hätte in Musik setzen wollen, keine komische, sondern eine sehr ernste Oper daraus geworden wäre, was eben nicht seine Absicht war. [...] Die Oper sollte eine komische sein; Berlioz machte daher auch darin Konzessionen, dass er die Tradition der Pariser komischen Oper festhielt und den Dialog zwischen den einzelnen Gesangsnummern beibehielt. »: notre traduction. Richard Pohl, Beatrice und Benedikt, Hektor Berlioz, p. 185.

6 Ibid., p. 184 et 189.

7 Ibid., p. 193. Notre traduction: «Hier folgt eine Szene voll drastischer Komik, in welcher Berlioz seinem Herzen einmal Luft gemacht und sich als Dichter mit treffender Ironie für den Ärger und die Plagen revanchiert hat, die er als Komponist und Dirigent so oft zu erdulden hatte. »

8 Ibid., p. 194. «Berlioz erzählte mir mit Lachen, dass ein jetzt in Paris lebender deutscher Kontrapunktist diese durchaus korrekte Fuge für das beste Stück in seiner Oper erklärt, die Ironie also gar nicht bemerkt habe!"

9 "Hektor Berlioz, Franz Liszt, Richard Wagner, - diese drei Namen bilden eine zusammenhängende Reihe von reformatorischen Thaten, welche mit Beethovens Tode beginnen, bis in die Gegenwart und noch darüber hinausreichen. [...]": notre traduction. R. Pohl, Einführung, Hektor Berlioz, p. XIII.

10 «Der Text schildert in poetischer Sprache die Reize der Natur, wie sie sich vor den Augen einer liebenden Braut entfalten, die in Erwartung ihres Glückes still erbebt. »: notre traduction. R. Pohl, Beatrice und Benedikt, Hektor Berlioz, p. 196.

11 "Es muss bei dieser Gelegenheit mit besonderer Anerkennung die vorzüglische Bearbeitung des französischen Textes durch R. Pohl erwähnt werden. Sie übertrifft das französische original 
an poetischem Werthe, und Pohl hat vieles in demselben nur angebeutete erst in das reich poetischer Anschauung hinübergehoben." notre traduction. Neue Zeitschrift für Musik, Heinrich Borges, 17 avril 1863, pp. 135-136.

12 Une biographie de Rosa von Milde et de son mari Feodor, également chanteur, a été réalisée par leur fils: Von Milde, F. (1918). Ein ideales Künstlerpaar. Rosa und Feodor von Milde / Ihre Kunft und ihre Zeit. Leipzig: Breitkopf \& Härtel. 